\title{
SURGICAL MANAGEMENT OF CHRONIC RHINOSINUSITIS. ANALYTICAL REVIEW
}

\author{
Sergey Kosyakov, Ekaterina Pchelenok \\ Department of ENT, National Medical Academy of Continuous Professional Education, \\ Russian Federation
}

\begin{abstract}
The modern opinion of chronic rhinosinusitis (CRS) treatment is described in this article from the viewpoint of evidential medicine. There is a review of researches concerning CRS medical therapy and evidence for surgery management is given. Functional endoscopic sinus surgery (FESS) is widely used today in CRS treatment but the more recent researches have shown that FESS does not ensure absolute recovery. A comprehensive treatment paradigm should entail medical therapy to control inflammation and infection and targeted surgery when indicated in medically recalcitrant cases. Surgery does not represent a cure to CRS but rather a key intervention in the overall management paradigm of CRS. Scr Sci Med. 2018;50(1):9-14
\end{abstract}

Keywords: chronic rhinosinusitis, endoscopic sinus surgery, medical therapy

Over the past few decades, surgery for chronic rhinosinusitis (CRS) has undergone progressive changes with the introduction of new technologies. In Russia we started to use the functional endoscopic sinus surgery (FESS) 25 years ago and we were very optimistic when looking to the future. However, now is the time to revise what we are doing as many surgical techniques are still debatable. Surgical indications for CRS rely on accurate diagnosis and first and foremost thorough attempts at medical management. It is very important to find the correlation between patients' complaints (subjective signs) and objective signs (mucosal inflammation, blockage of the natural ostium, state of the ostiomeatal complex and

Address for correspondence:

Sergey Kosyakov

National Medical Academy of Continuous Professional

Education

7 Ivankovskoe St

125367 Moscow

Russian Federation

e-mail:epchelenok@yandex.ru,serkosykov@yandex.ru

Received: August 7, 2017

Accepted: March 23, 2018 obtaining of bacterial culture for proper antibiotic prescription). The most common indications for FESS regarding CRS are as follows: chronic sinusitis refractory to medical treatment, recurrent sinusitis, nasal polyposis, antrochoanal polyps, sinus mucoceles, foreign body removal.

Computed tomography (CT) is considered the golden standard in diagnosis of CRS and its timing is of vital importance (during the period of exacerbation or in remission). However, the specificity of CT is very low - about $2 \%$ (1). This is why we consider it only as a surgical road map for the identification of the anatomical structures, which can play a role in the pathogenesis of chronic sinusitis. The status of mucosa is sometimes very difficult to estimate by CT.

The decision to operate on the patient must be taken only when all medical therapies have failed, or there are serious preclusions of the medical management (serious deviation of the nasal septum with reduction of air flow, threat of complications, and serious nasal obstruction with polyps). In some cases medical treatment may not be needed e.g. when there is a foreign body in the maxillary sinus with micetoma. 
For the evidence-based medical treatment we can use: nasal saline irrigations, short courses of systemic antibiotics and topical treatments in cases of acute bacterial complications (2-4). Long courses of low-dose macrolides can be used in some groups of patients (5-7). Topical corticosteroids are used because of their universal anti-inflammatory activity $(1,8,9)$. The main problem with intranasal corticosteroids (INCS) administration, however, is the guarantee to enrich the sinus mucosa in an appropriate quantity. Also one of the FESS goals is to provide the patency of the sinus ostium but at the same time we need special INCS delivery devices for the sinuses.

When the patient meets the criteria for surgery, we can use several approaches FESS, minimally invasive surgical therapy (MIST), maximal techniques, and Balloon sinus surgery. What may be of great concern about the last technology is that the surgical indications were not defined for it and there exist no validated symptom measurement tools yet (10).

MIST was proposed in 1996 as the cure for pathologic disease with conservative approach. MIST is transition space surgery allowing reestablishment of ventilation and drainage through natural sinus ostia without touching the larger sinuses. The advantages of this surgery are less mucosal stripping, less complications of bone exposure, minimal mucosal scarring, improved disease resolution, reduced time consumption and minimal postoperative care. This is not recommended for severe cases because it does not prevent the mucosal remodeling in CRS (11).

Remodeling in the sinuses can lead to potential irreversible structural changes and thus poses a theoretical threat to the current management model of CRS. If we compare sinus disease with pneumonia and sinus with lungs, simple ventilation of the sinuses is thus unlikely to be an adequate treatment of CRS. In 1992, Kobayashi T and Baba S. showed the lack of antibiotic penetration within maxillary sinus of non-operated patients undergoing a course of nebulized antibiotics (12). FESS is better suited to allow access for topical preparations (13). Steroids have a theoretical potential to reverse remodeling through the ability to reverse pathologically remodeled airways by decreasing collagen deposition in the subepithelial basement membrane. However, there exists the possibility that steroids may delay or modify the remodeling process through anti-inflammatory actions. Thus surgery serves as an adjunct to maximum medical therapy, a concept that now includes the use of topical medical therapies (14).

The concept of FESS is predicated by the principle of reversible mucosal disease and the principle of maintaining patency of the ciliated respiratory epithelium flow pathways that constitute the optimal physiological condition of healthy sinuses. FESS contributes to the belief that the majority of pathologically remodeled mucosa is reversible to a more physiologic status. In fact this is contrary to the surgical results. The limited available histological studies suggest that despite clinical improvement, electronic microscopy continues to demonstrate irreversible mucosal changes after surgery. This is why maximal extensive surgical techniques are advocated for patients with severe diseases or dysfunctional sinus. A dysfunctional sinus is defined as one that has apparently lost its mucociliary function despite maximum medical treatment and surgery achieving adequate sinus ventilation. FESS involves the enlargement of ostia and the removal of polypoid disease, bony partitions and osteitic bone. Other surgical maneuvers include management of the middle and inferior turbinates or septum. Surgery does not represent a cure to CRS. The goals of surgery in CRS are merely to establish ventilation and facilitate drainage from the paranasal sinuses and to optimize the topical delivery of medications directly to the sinonasal mucosa. It is a generally supported concept that FESS improves symptoms and the quality of life in adult patients with CRS. A prospective study by Metson, Glicklicich (108 patients undergoing FESS) found significant improvement in symptoms and reduction in medication usage in $82 \%$ of the patients 1 year postoperatively (15). Some studies have shown consistent improvement, even as long as 10 years after surgery. There are however no direct comparative studies between minimally invasive techniques and traditional FESS. It has been shown that FESS has decreased morbidity and improved the results over the Caldwell-Luc procedures. Although not directly compared, it would seem that most minimally invasive and FESS outcome studies report similar overall results in short to mid-term follow-up.

A recent prospective, multi-institutional study compared medical versus surgical therapy for CRS. 
A total of 108 patients were prospectively enrolled into a nonrandomized, multi-institutional cohort. Patients elected continuous medical management or FESS and were followed to a primary end point of 6 months. Surgical patients reported significantly greater improvement than medically managed patients, based on validated quality of life questionnaires. Furthermore, surgical patients reported reduced usage of oral antibiotics and steroids and fewer missed days of work following FESS. But we can look at the results of such studies from a different angle. The decision to undergo the intervention is very difficult for the patient, particularly for men. Notably in rural areas, out of the big cities the patient has to make a decision in more advanced cases due to fewer alternative choices or they would present to the doctor in an emergency situation (e.g. complications). In most cases a patient's decision is based on one hand on the lack of satisfaction from the medical treatment, and on the other hand on the motivation of the practicing surgeon. Of course when patients overcomes the stress of the waiting period and finally arrive at the actual day of surgery they may feel very euphoric. This feeling is so strong, that many patients (men) reported that their sexual activity rises at that time (16).

A retrospective review of a cohort of prospectively enrolled patients with CRS who have undergone endoscopic sinus surgery, demonstrated mean response scores regarding sexual activity in the preoperative and postoperative period, which were significantly different (14). The patients are happy that they are alive and hope they would feel an improvement. Their nasal pack is removed (in fact the most boring of the patients experiences) and they are under thorough postoperative care (about one and a half months). Twice a day they go through suction and nasal irrigation while in hospital (usually performed by an attractive lady doctor) and they feel very happy. After the discharge from the hospital they continue to irrigate their nose, use topical medicines and, sadly, remember the bill from the doctor. They are afraid to admit to themselves that the situation is far from ideal. Therefore, this becomes an important factor. It is to be established how many of the patients are men and how long they feel an improvement after the surgery. The different standpoint can be demonstrated by the study of quality of life out- comes after functional endoscopic sinus surgery (17). The authors aim to demonstrate the evidence supporting the efficacy of FESS to improvement of longterm quality of life outcomes in patients with CRS. It must be noted however that several factors have been shown to deteriorate results, for example ASA triad disease sufferers, female gender, non-Caucasian ethnicity. Patients of male gender have been shown to have a slightly higher baseline quality of life (QoL) than the average CRS patient.

Another prospective study directly evaluated the effect of FESS on antibiotic utilization. A total of 503 patients were followed for an average of 17.2 months. These patients reported $57.2 \%$ reduction in the length of time of antibiotics administration following FESS (remember the placebo effect which can exceed $40 \%)(2,16,18,19)$.

However not all published evidence advocates the usefulness of FESS for CRS. A recent Cochrane review concluded that FESS as currently practiced is a safe surgical procedure. The limited evidence available suggests that FESS does not confer additional benefit to the medical treatment and is not superior to medical treatment in patients with CRS in a long follow-up. This included three randomized controlled trials. The first compared endoscopic antrostomy to more traditional inferior meatal antrostomy (an important consideration for Russia due to tradition and long history of inferior antrostomy). Another study showed a relapse rate of $2.4 \%$ in the FESS and sinus irrigation group compared to $5.6 \%$ in the sinus irrigation only group. There is no consensus on the definition of maximum medical therapy (20).

To study the outcomes of FESS in CRS is a difficult task because of the lack of pre-set homogeneous clinical groups. CRS is a group of diseases with multifactorial pathogenesis. Another point of interest is the size of the surgical antrostomy initially centered on the uncertain role of nitric oxide within the sinus considering that normal concentrations of nitric oxide affect ciliary function and provide antimicrobial properties.

It is possible to find many similarities between sinusitis and otitis media. There is a respiratory zone (nasal cavity with the osteomeatal complex and tympanic cavity) and a pulmonary zone (sinuses and mastoid). The gas exchange in the mastoid has 
been studied thoroughly. If we extrapolate this data to sinuses, we can assume that the mechanism is the same, but with a difference in size and rigidity. There are few studies on this topic. One of them demonstrates how changes occur in the additional ostium nitric oxide concentration. For therapeutic purposes, however, the larger antrostomy is better in terms of endoscopic control and medicine delivery.

Surgery can fail for many different reasons. Common causes of suboptimal results include recurrent inflammation and poliposis, adhesions or synechiae formation, middle turbinate lateralization and stenosis of the surgically enlarged sinus ostia. Controlling of these postoperative issues has been shown to lead to better long-term outcomes. The primary goals of early postoperative care are to reduce mucosal inflammation and infection, improve short-term patient symptoms, promote early return of ciliary function and prevent complications. Among common postoperative interventions there are nasal saline irrigations, which aid debris removal, softening of crusting and improvement of mucociliary clearance. Recently about 6 randomized studies have evaluated the impact of irrigations on clinical outcomes of FESS and experts agree that large-volume irrigations should be started 24 to 48 hours after FESS (21-26). The sinus cavity following FESS has a large amount of crusting, old blood and retained secretions. This localized status provides the framework for scarring, ostial stenosis and middle turbinate lateralization. Debridement of the postoperative sinus optimizes early mucosal healing by reducing the inflammation. Debridement includes endoscope usage with suction for soft debris. Most experts agree that optimal debridement must be done on the first week after FESS.

Topical nasal corticosteroids are an integral component of anti-inflammatory CRS medical therapy. Application techniques include nasal sprays with approved topical steroids but nasal sprays tend to provide more nasal coverage. We need improved sinus penetration. We require new delivery systems. The 3 most recent level $1 \mathrm{~b}$ trials for postoperative topical steroid sprays demonstrated a significant improvement following FESS. They also demonstrated the need to start topical nasal steroids between the first 2 and 6 weeks after surgery (27-29).
Patients undergoing FESS for medically recalcitrant CRS have significant underlying mucosal inflammation and experts recommend the short course protocols using prednisolone $30 \mathrm{mg}$ for 4 days, followed by $20 \mathrm{mg}$ for 4 days and completing the course with $10 \mathrm{mg}$ for 4 days. The total duration is therefore 12 days with a cumulative dose of $240 \mathrm{mg}$. A level $1 \mathrm{~b}$ study (Wright and Agrawal) recommend perioperative systemic steroids $30 \mathrm{mg} 5$ days before FESS and 9 days after FESS (14 days, a cumulative dose of 420 mg) $(30,31)$.

A very promising method for direct delivery to the sinus in an appropriate dose is the drug-eluting middle meatal stents. The Propel implant (Intersect ENT, Palo Alto, CA, USA) is a dissolvable mometasone furoate-eluting stent, which is placed into the dissected ethmoid cavity and expands to contact the mucosa. The drug-eluting stent is composed of polylactide-co-glycolide. This polymer is a common biomedical substance which is used in many types of suture materials and as shown in prior animal studies, does not incite the inflammatory response (32). A total dose of $370 \mu \mathrm{g}$ of mometasone fuorate (MF) is blended into the polymer structure of polylactideco-glycolide, which releases the MF by diffusion in a controlled fashion over approximately 30 days. To prevent confounding effects, patients are not permitted to use either topical or systemic steroids for 30 days following FESS (33).

Two randomized trials (1b) evaluating the stent demonstrated a reduction in the need for postoperative interventions, lysis of adhesions, courses of oral steroids, as well as reduction in polyp recurrence (16, 32). The eluting stents may play an integral role in postoperative care following FESS. But the future studies need to evaluate the role of these devices in different groups of patients with CRS.

A comprehensive treatment paradigm should entail medical therapy to control inflammation and infection and targeted surgery when indicated in medically recalcitrant cases. Surgery does not represent a cure to CRS but rather a key intervention in the overall management paradigm of CRS.

\section{REFERENCES}

1. Hwang PH, Irwin SB, Griest SE, Caro JE, Nesbit GM. Radiologic correlates of symptom-based diagnostic criteria for chronic rhinosinusitis. Oto- 
Sergey Kosyakov, Ekaterina Pchelenok

laryngol Head Neck Surg. 2003;128(4):489-96. doi: 10.1016/S0194-59980223295-7.

2. Bachmann G, Hommel G, Michel O. Effect of irrigation of the nose with isotonic salt solution on adult patients with chronic paranasal sinus disease. Eur Arch Otorhinolaryngol.2000; 257(10):537-41.

3. Harvey R, Hannan SA, Badia L, Scadding G. Nasal saline irrigations for the symptoms of chronic rhinosinusitis. Cochrane Database Syst Rev. 2007; (3):CD006394. doi: 10.1002/14651858.CD006394. pub2.

4. Taccariello M, Parikh A, Darby Y, Scadding G. Nasal douching as a valuable adjunct in the management of chronic rhinosinusitis. Rhinology. 1999:37(1): 29-32.

5. Desrosiers MY, Salas-Prato M. Treatment of chronic rhinosinusitis refractory to the treatments with topical antibiotic therapy delivered by means of a large-particle nebulizer: results of a controlled trial.Otolaryngol Head Neck Surg. 2001;125(3):265-9. doi: $10.1067 / \mathrm{mhn} .2001 .117410$.

6. Videler WJ, van Drunen CM, Reitsma JB,Fokkens WJ. Nebulized bacitracin/colimycin: a treatment option in recalcitrant chronic rhinosinusitis with Staphylococcus aureus? A double-blind, randomized, placebo-controlled, cross-overpilot study. Rhinology. 2008;46(2):92-8.

7. Uren B, Psaltis A, Wormald PJ. Nasal lavage with mupirocin for the treatment of surgically recalcitrant chronic rhinosinusitis. Laryngoscope. 2008;118(9):1677-80. doi: 10.1097/ MLG.0b013e31817aec47.

8. Cannady SB, Batra PS, Citardi MJ, Lanza DC. Comparison of delivery of topical medications to the paranasal sinuses via 'vertex-to-floor' position and atomizer spray after FESS. Otolaryngol Head Neck Surg. 2005;133(5):735-40. doi: 10.1016/j. otohns.2005.07.039.

9. Snidvongs K, Kalish L, Sacks R, Craig JC, Harvey RJ. Topical steroid for chronic rhinosinusitis without polyps. Cochrane Database Syst Rev. 2011; (8):CD009274. doi: 10.1002/14651858.CD009274.

10. Ramakrishnan VR, Kennedy D. Advances in the surgical management of rhinosinusitis and nasal polyposis. Curr Allergy Asthma Rep. 2011; 11(3):220-9. doi: 10.1007/s11882-011-0182-y.

11. Chiu AG, Kennedy DW. Disadvantages of minimal techniques for surgical management of chronic rhinosinusitis. Curr Opin Otolaryngol Head Neck Surg. 2004;12(1):38-42.

12. Kobayashi T, Baba S. Experimental study on intramaxillary injection of fosfomycin. Effects of fosfomycin on experimental acute sinusitis in rabbits. Jpn J Antibiot. 1992;45(10):1319-31.

13. Bassiouni A, Chen PG, Wormald PJ. Mucosal remodeling and reversibility in chronic rhinosinusitis. Curr Opin Allergy Clin Immunol. 2013;13(1):412. doi: 10.1097/ACI.0b013e32835ad09e.

14. Benninger MS, Khalid AN, Benninger RM, Smith TL. Surgery for chronic rhinosinusitis may improve sleep and sexual function. Laryngoscope. 2010;120(8):1696-700. doi: 10.1002/lary.21010.

15. Sedaghat AR, Metson R, Gray ST. Impact of day of week on outcomes of endoscopic sinus surgery for chronic rhinosinusitis. Am J Rhinol Allergy. 2015; 29(5):378-82. doi: 10.2500/ajra.2015.29.4228.

16. Smith TL, Litvack JR, Hwang PW, Loehrl TA, Mace JC, Fong KJ, et al. Determinants of outcomes of sinus surgery: a multiinstitutional prospective cohort study. Otolaryngol Head Neck Surg. 2010; 142(1):55-63. doi: 10.1016/j.otohns.2009.10.009..

17. Soler ZM, Smith TL. Quality of life outcomes after functional endoscopic sinus surgery. Otolaryngol Clin North Am. 2010;43(3):605-12. doi: 10.1016/j. otc.2010.03.001

18. Mace J, Yvonne ML, Carlson NE, Litvack JR, Smith TL. Effects of depression on quality of life improvement after endoscopic sinus surgery. Laryngoscope. 2008;118(3):528-34. doi: 10.1097/ MLG.0b013e31815d74bb.

19. Soler ZM, Mace J, Smith TL. Fibromyalgia and chronic rhinosinusitis: outcomes after endoscopic sinus surgery. Am J Rhinol. 2008;22(4):427-32. doi: 10.2500/ajr.2008.22.3198.

20. Khalil HS, Nunez DA. Functional endoscopic sinus surgery for chronic rhinosinusitis. Cochrane Database Syst Rev. 2006;(3):CD004458. doi: 10.1002/14651858.CD004458.pub2.

21. Freeman SR, Sivayoham ES, Jepson K, de Carpentier J.. A preliminary randomised controlled trial evaluating the efficacy of saline douching following endoscopic sinussurgery. Clin Otolaryngol. 2008; 33(5):462-5. doi: 10.1111/j.1749-4486.2008.01806.x.

22. Pinto JM, Elwany S, Baroody FM, Naclerio RM. Effects of saline sprays on symptoms after endoscopic sinus surgery. Am J Rhinol. 2006;20(2):191-6. 
23. Fooanant S, Chaiyasate S, Roongrotwattanasiri K. Comparison on the efficacy of dexpanthenol in sea water and saline in postoperative endoscopic sinus surgery. J Med Assoc Thai. 2008; 91(10):1558-63.

24. Liang KL, Su MC, Tseng HC, Jiang RS. Impact of pulsatile nasal irrigation on the prognosis of functional endoscopic sinus surgery. J Otolaryngol Head Neck Surg. 2008;37(2):148-53.

25. Pigret D, Jankowski R. Management of post-ethmoidectomy crust formation: randomized singleblind clinical trial comparing pressurized seawater versus antiseptic/mucolytic saline. Rhinology. 1996;34(1):38-40.

26. Staffieri A, Marino F, Staffieri C, Giacomelli L, D'Alessandro E, Maria Ferraro S, et al. The effects of sulfurous-arsenical-ferruginous thermal water nasal irrigation in wound healing after functional endoscopic sinus surgery for chronic rhinosinusitis: a prospective randomized study. Am J Otolaryngol. 2008; 29(4):223-9. doi: 10.1016/j.amjoto.2007.07.002.

27. Bhalla RK, Payton K, Wright ED. Safety of budesonide in saline sinonasal irrigations in the management of chronic rhinosinusitis with polyposis: lack of significant adrenal suppression. J Otolaryngol Head Neck Surg. 2008; 37(6):821-5.

28. Del Gaudio JM, Wise SK. Topical steroid drops for the treatment of sinus ostia stenosis in the postoperative period. Am J Rhinol. 2006;20(6):563-7.

29. Welch KC, Thaler ER, Doghramji LL, Palmer JN, Chiu AG. The effects of serum and urinary cortisol levels of topical intranasal irrigations with budesonide added to saline in patients with recurrent polyposis after endoscopic sinus surgery. Am J Rhinol Allergy. 2010; 24(1):26-8. doi: 10.2500/ ajra.2010.24.3418.

30. Rudmik L, Smith T. Evidence-based practice. Postoperative care in endoscopic sinus surgery. Otolaryngol Clin N Am. 2012; 45(5):1019-32. doi: 10.1016/j.otc.2012.06.006.

31. Wright ED, Agrawal S. Impact of perioperative systemic steroids on surgical outcomes in patients with chronic rhinosinusitis with polyposis: evaluation with the novel Perioperative Sinus Endoscopy (POSE) scoring system. Laryngoscope. 2007 Nov;117(11 Pt 2 Suppl 115):1-28. doi: 10.1097/ MLG.0b013e31814842f8.

32. Lund VJ, Flood J, Sykes AP, Richards DH. Effect of fluticasone in severe polyposis. Arch Otolaryngol Head Neck Surg. 1998;124(5):513-8.
33. Li PM, Downie D, Hwang PH. Controlled steroid delivery via bioabsorbable stent: safety and performance in a rabbit model. Am J Rhinol Allergy. 2009; 23(6):591-6. doi: 10.2500/ajra.2009.23.3391. 